\title{
The Association between Self-Reported Major Life Events and the Presence of Uterine Fibroids
}

\author{
Anissa I. VINES, MS, PhD \\ Department of Epidemiology Gillings School of Global Public Health University of North Carolina \\ at Chapel Hill 266 Rosenau Hall, CB\#7435 Chapel Hill, NC 27599-7435
}

Myduc TA, PhD

Department of Epidemiology, CB\#7435 Gillings School of Global Public Health University of North Carolina at Chapel Hill myduc@email.unc.edu

Denise A. ESSERMAN, PhD

Department of Medicine School of Medicine University of North Carolina at Chapel Hill 5034B Old Clinic Building, CB\#7228 Chapel Hill, NC 27599-7228 919-843-2887 Fax: 919-843-4031

Denise_Esserman@med.unc.edu

\section{Abstract}

Purpose-Uterine fibroids are the most common benign tumors in reproductive age women. Factors associated with condition such as psychosocial stress are still being elucidated. This paper explores the association between major life events (MLE) stress and fibroids.

Methods-Prevalence ratios (PR) and 95\% confidence intervals (CI) were used to determine the association between MLE stress (number of events and stress intensity) and fibroids in 556 black and 373 white women in the Uterine Fibroid Study, 1996-1999.

Main Findings-Fibroids were prevalent in $74 \%$ and $50 \%$ of the black and white women, respectively. The mean number of MLE reported by each race group was 2 . Among white women, the PR for those who reported at least 1 event compared to those with no events were significant after adjusting for age and study identified fibroid risk factors $[\mathrm{PR}(1$ and 2 events $)=1.7 ; 95 \% \mathrm{CI}$ : $(1.2,2.5)]$, [PR(3 events $)=1.9 ; 95 \%$ CI: $(1.3,2.7)]$, and [PR $(4$ events $)=1.5 ; 95 \%$ CI: $(1.0,2.1)]$, respectively. At all levels of stress compared to no events, significant associations with fibroids were found among the white women. For black women, the PR for fibroids was only significant in the high stress group compared to those without an experienced event, after adjusting for fibroid risk factors $[\mathrm{PR}=1.2 ; 95 \% \mathrm{CI}=(1.1,1.4)]$.

Conclusion-Examining the number and stress intensity of MLE enriched our understanding of this stressor and fibroids. Further research is needed to understand the role of stress on fibroids among black women.

(C) 2010 Jacobs Institute of Women's Health. Published by Elsevier Inc. All rights reserved 919-843-3539 919-966-7457 Fax: 919-966-2089 avines@email.unc.edu.

Publisher's Disclaimer: This is a PDF file of an unedited manuscript that has been accepted for publication. As a service to our customers we are providing this early version of the manuscript. The manuscript will undergo copyediting, typesetting, and review of the resulting proof before it is published in its final citable form. Please note that during the production process errors may be discovered which could affect the content, and all legal disclaimers that apply to the journal pertain. 


\section{Introduction}

Uterine fibroids are the most common benign tumors in women of childbearing age. The morbidity associated with this gynecologic condition is high with fibroids affecting the quality of life of about $30 \%$ of reproductive age women and many experience abnormal bleeding, severe pelvic pain, fatigue, activity limitation, and urinary and bowel problems (Haney, 2000; Kjerulff, et al., 1996; Walker \& Stewart, 2005). As with many other chronic conditions, black women have a risk of fibroids that is two to three times higher than that of white women (Baird et al., 2003; Baird, 2004; Marshall et al., 1997; Parazzini et al., 1988). This racial disparity in uterine fibroid prevalence and morbidity has not been sufficiently explored in studies to date. Subsequently, the etiology and some risk factors for fibroids smoking, parity, and obesity - remain unclear. Another factor that has not been fully explored in understanding uterine fibroids is psychosocial stress.

Stress from major life events has been examined for decades as a risk factor for poor physical and mental health outcomes. This form of stress occurs as a result of "acute changes which require major behavioral readjustments within a relatively short period of time (e.g. birth of first child; divorce)" (Thoits, 1995). There is evidence indicating that stress can increase disease risks when stressful events are perceived as insurmountable and when coping strategies are unable to buffer the threat or demand (Cohen \& Williamson, 1991). It is also biologically plausible that stress can lead to fibroids as a result of fluctuations in estrogen and progesterone hormone levels, both important in fibroid development, caused by the stress activation of the hypothalamic pituitary adrenal axis and the subsequent release of cortisol, a stress hormone (Nepomnaschy et al., 2004). To date, only one study has examined stress in relation to uterine fibroids. In that study, an increased risk of fibroids resulting from perceived racial discrimination was reported (Wise et al., 2007).

Given the biological plausibility of stress and fibroids and the sparse literature in this area, the goals of this study were to provide insight into the association between major life events stress and uterine fibroids separately in black and white women by addressing the following 2 research aims: (1) to determine if the experience of more major life events is associated with the presence of fibroids; and (2) to examine the stress intensity of the life events experienced in association with the presence of uterine fibroids.

\section{Methods}

The National Institute of Environmental Health Sciences Uterine Fibroid Study (UFS) was conducted from 1996-1999 in the Washington, D.C. area in collaboration with the George Washington University Medical Center. The goal of the UFS was to estimate the agespecific cumulative incidence of uterine fibroids and to assess risk factors for fibroids. Detailed methods have been described previously (Baird et al., 2003). Briefly, the computerized membership records of a prepaid health plan in Washington, DC, were used to randomly select women aged 35-49 years. Those selected were screened for eligibility by telephone interview. Eligibility criteria were as follows: 1) the computerized listing had correctly identified a current member aged 35-49 years, and 2) a telephone interview could be conducted in English. Initial enrollment and screening involved 1,430 eligible women with a response rate of about $80 \%$. Of the 1,143 premenopausal women screened, the majority (over 90\%) self-identified into either the black or the white non-Hispanic race groups, and the reported results are limited to these two groups. We excluded 156 women (14\%) because they did not complete the self-administered questionnaire, the source of the life events data. We also excluded 53 women ( 34 black, 19 white) because of missing data on fibroid status. The final sample size for the analysis was 929 . 
Human Subjects Review Boards at the National Institute of Environmental Health Sciences and the George Washington University Medical Center reviewed the initial study, and participants gave informed consent. For this analysis of life events stress and uterine fibroids, the University of North Carolina at Chapel Hill Human Subject Review Board reviewed and approved this project.

Fibroid status-All women in the study had ultrasound evidence - radiology records from a recent pelvic ultrasound examination or a study provided ultrasound - to document the presence of a uterine fibroid(s). Self-report of "no fibroids" was not accepted because undiagnosed fibroids were common (about half of the undiagnosed women in our study were found to have fibroids at ultrasound screening) (Baird et al., 2003).

Major life events—Using a self-administered questionnaire, respondents were asked about their experience of 14 major life events occurring within the past 12 months. The life events - self job loss; family member job loss; self/family member looking for new job, self new job; family member new job; self major accident, operation or illness; close friend/ relative major accident; operation or illness; new romantic relationship; serious problems in marriage or other close relationship; divorce or breakup of primary relationship; significant loss of spendable income; changed/moved residence; and robbery or assault — included items comparable to those on the Holmes and Rahe life events scale (Holmes \& Rahe, 1967). For each event, respondents indicated if they had experienced the event (i.e. yes $=1 /$ no $=0)$ and their stress appraisal of each event ( $1=$ not at all stressful; $2=$ mildly stressful; $3=$ moderately stressful; $4=$ =severely stressful). The number of life events was summed and the total was categorized into 4 groups: no event, 1 event, 2 events, 3 events, and 4 or more events. A stress intensity variable was also calculated by multiplying the experience of a life event (yes, no) and the stress appraisal of the event. The resulting distribution was divided into 4 categories of stress intensity: None; Low; Medium; High. The category 'None' was comprised of women who did not experience any of the life events (19.6\% and $21.5 \%$ black and white women). The remaining categories (i.e. low, medium, and high) were based on $\sim 80 \%$ of the data and determined by tertiles of the distribution without those in the 'None' category. Due to missing values for some women who only answered yes/no to experiencing a life event, the number of women who did not experience any of the 14 life events does not equal the number of women in the 'none' category of life events stress intensity.

Study variables-Sociodemographical characteristics and other covariates came from either the Uterine Fibroid Study (UFS) telephone interview (occupation, age at menarche, parity), self-administered questionnaire (education, income, household size, marital status, race), or clinic visit (body mass index). The 2000 Census Occupational Groupings (Fronczek \& Johnson, 2003) was used to classify respondents into 2 occupational groups professional or managerial occupations and non-professional or non-managerial (i.e. service positions) - based on self-report of longest held occupation. Total reported household income was adjusted based on the total number of people supported by the income and indexed to a household size of 2. Adjusted household income was then categorized into 4 groups: < \$40,000 and/or Medicaid; $\$ 40,000-\$ 59,999 ; \$ 60,000-\$ 99,999$; and at least $\$ 100,000$. All potential risk factors for fibroids were determined a priori based on previous extensive analyses of the UFS study data and included the number of full-term pregnancies delivered after age 24 years, body mass index (BMI), age at menarche, and physical activity (Baird et al., 2007).

\section{Statistical analysis}

Descriptive statistics (proportions and frequencies for categorical variables; means and standard deviations (SD) for continuous data) were calculated for the outcome of interest 
(presence of uterine fibroids), exposures of interest (number of life events; life events stress intensity), and the a priori identified UFS study risk factors.

The relationship between major life events stress (number of events and stress intensity) and uterine fibroids was evaluated using Poisson regression with a robust variance estimator (Zou, 2004) to calculate prevalence ratios (PR) and 95\% confidence intervals. Because black women have a significantly higher risk of fibroids and the distributions of the examined socioeconomic variables by race, analyses were conducted separately for each race group. Progressive model building was used to assess the impact of potential risk factors and/or confounders. Wald statistics were used to determine the significance of each exposure of interest in the progressive models.

\section{Results}

Women in this study reported an average of about 2 major life events within the past 12 months with about $20 \%$ reporting no events at all (Table 1). Consistent with the uterine fibroid literature, black women had a higher prevalence of fibroids and tended to be diagnosed on average about a year earlier than white women. Majority of black women reported attaining some college or junior college training, had an adjusted annual household income of less than $\$ 40,000$, and were employed in non-professional or management positions. The reverse demographics were found among the white women with over $80 \%$ having at least a college degree, $41 \%$ with an adjusted household income ranging from $\$ 60,000$ to $\$ 99,999$, and $76 \%$ employed in a professional or managerial position.

The number of major life events was positively associated with fibroids, but the associations were stronger among the white women (Table 2). For white women, the prevalence of fibroids (Model 2) was 1.7 [95\% CI: 1.2, 2.4] times higher among those who reported one event and two events; 1.9 for those with 3 events [95\% CI: 1.3, 2.7] compared to those who had not experienced any of the study's 14 major life events. These findings attenuated slightly after adjusting for socioeconomic status and fibroid risk factors.

The stress intensity of major life events and fibroids yielded similar results (Table 3). Among the black women, there was a modest association between high stress intensity and fibroids (Model 2) that remained after adjusting for socioeconomic status (Model 4). The prevalence of fibroids was higher among white women with low or medium stress intensity (PR=1.8; 95\%CI: 1.2, 2.5) compared to those with no stress.

\section{Discussion}

Uterine fibroid (fibroids) research is growing, yet understanding psychosocial stress as a risk factor for this chronic gynecologic condition remains in its infancy. The purpose of this paper was to explore the association between major life events stress and fibroids. In doing so, we examined not only the number of major life events experienced, but also the stress intensity associated with the events in association with the presence of fibroids.

In this cross-sectional study, women reported on average 2 major life events within the past 12 months. A positive association was between the number of events and the prevalence of fibroids, the estimates were the strongest among the white women with adjustment for socioeconomic status slightly attenuating the effects. Stress intensity was also found to be associated with fibroids among the white women at all levels of stress compared to those without an experienced event. We only found an association with the presence of fibroids among the black women in the high stress intensity group. These findings are consistent with Wise and colleagues who also reported a relationship between stress and uterine fibroids (Wise et al., 2007). 
Although the findings reported in this paper are based on cross-sectional data and the timing of fibroid onset is still to be understood, this is the first study to consider the contribution of major life events stress on the presence of fibroids. With the plausibility of stress causing fluctuations in hormonal levels resulting from the arousal of the hypothalamic pituitary adrenal axis (HPA-axis) (Nepomnaschy et al., 2004), our findings support the need for additional research examining not only the association between psychosocial measures and fibroids but also the exploration of the biological mechanisms underlying the hypothesized association.

We were able to examine the association of life events stress on the presence of fibroids in a diverse, representative group of health plan participants where information on disease status was based on ultrasound data. The racial disparity in the prevalence of fibroids was supported by the study data. The data provided an opportunity to look at the stress-fibroid association within racial groups to account for the variation in distributions of important fibroid risk factors and socioeconomic variables. Another strength is that the study participants were asked to report specifically on 14 major life events experienced and the associated perceived stress related to each life event. Although we speculated that the prevalence of fibroids would be greater among black women due to the higher likelihood of them experiencing more life events stress as a result of being disproportionately represented in the low socioeconomic stratum, this was not supported by the data. Again, it may be that the 14 life events queried as part of the Uterine Fibroid Study did not fully capture the major life events for black women. For example, discrimination disproportionately affects black women and was previously shown to be associated with fibroids (Wise et al., 2007); however, the measure on life events did not include any discriminatory events

While the goal of this study was to assess the strength of association between life events stress and the presence of fibroids, the effect sizes observed were modest, especially among the black women which suggest the potential for residual confounding given the selection of covariates. However, covariates included in the analyses were selected a priori based on the results from the extensive analyses of the data. For example, contraception is a variable that is often included in studies pertaining to women's health, especially hormone sensitive conditions. However, prior analyses of the data did not find contraception to be associated with fibroids in this study population. As a result, the potential for residual confounding due to the omission of variables was minimized.

The prevalence of uterine fibroids among reproductive age women is high and the associated morbidity can cause declines in overall quality of life by: causing fertility problems; affecting work and social activities because of pain and heavy bleeding; and leading to mental distress related to the management of the disease symptoms. We assessed the robustness of our findings by excluding women who reported debilitating fibroid symptoms and did not observe a change in the estimates. It is important to point out that although we saw an association between life events stress and fibroids, the presence of an increasing trend or dose-effect was not evident. Further work on life events stress and fibroids needs to be done to improve our understanding of this chronic condition and bring attention to the potential role of stress on fibroids.

\section{Acknowledgments}

Dr. Anissa Vines' work on this project was supported by the National Institute of Child Health and Human Development (RO3-MH61057) and the University of North Carolina at Chapel Hill, BIRCWH Program, K12HD001441-06. Dr. Myduc Ta's work on this project was provided by the National Institute of Child Health and Human Development, RO3-MH61057 (awarded to AI Vines). Dr. Denise Esserman's support was provided by a Translational Science Award, UL1 RR025747 to the University of North Carolina at Chapel Hill. The authors report no known financial conflict of interest. Dr. Vines had full access to all the data in the study and takes 
responsibility for the integrity of the data and the accuracy of the data analysis. The authors would like to acknowledge Dr. Donna D. Baird, senior epidemiologist at the National Institute of Environmental Health Sciences for the use of the Uterine Fibroid Study data. The authors thank Thu Thi Xuan Nguyen and Monica Hadley for their considerable aid in finalizing this work for publication. Data for this project would not have been possible without the expertise and committed staff of Social and Scientific Systems, Inc. (formerly CODA, Inc.), Research Triangle Park, NC and the participants in the Uterine Fibroid Study.

\section{References}

Baird DD. Invited commentary: Uterine leiomyomata-we know so little but could learn so much. American Journal of Epidemiology 2004;159(2):124-126. [PubMed: 14718212]

Baird DD, Dunson DB, Hill MC, Cousins D, Schectman JM. Association of physical activity with development of uterine leiomyoma. American Journal of Epidemiology 2007;165(2):157-163. [PubMed: 17090618]

Cohen S, Williamson GM. Stress and infectious disease in humans. Psychological Bulletin 1991;109(1):5-24. [PubMed: 2006229]

Baird DD, Dunson DB, Hill MC, Cousins D,D, Schectman JM. High cumulative incidence of uterine leiomyoma in black and white women: Ultrasound evidence. American Journal of Obstetrics and Gynecology 2003;188(1):100-107. [PubMed: 12548202]

Fronczek, P.; Johnson, P. Occupations 2000: A census 2000 brief. 2003. Retrieved October 06, 2008, from www.census.gov/hhes/www/ioindex/reports.html

Haney AF. Clinical decision making regarding leiomyomata: What we need in the next millennium. Environmental Health Perspectives 2000;108(Suppl 5):835-839. [PubMed: 11035991]

Holmes TH, Rahe RH. The social readjustment rating scale. Journal of Psychosomatic Research 1967;11(2):213-218. [PubMed: 6059863]

Kjerulff KH, Langenberg P, Seidman JD, Stolley PD, Guzinski GM. Uterine leiomyomas - racial differences in severity, symptoms and age at diagnosis. Journal of Reproductive Medicine 1996;41(7):483-490. [PubMed: 8829060]

Marshall LM, Spiegelman D, Barbieri RL, Goldman MB, Manson JE, Colditz GA, et al. Variation in the incidence of uterine leiomyoma among premenopausal women by age and race. Obstetrics and Gynecology 1997;90(6):967-973. [PubMed: 9397113]

Nepomnaschy PA, Welch K, McConnell D, Strassmann BI, England BG. Stress and female reproductive function: A study of daily variations in cortisol, gonadotrophins, and gonadal steroids in a rural mayan population. American Journal of Human Biology: The Official Journal of the Human Biology Council 2004;16(5):523-532. [PubMed: 15368600]

Parazzini F, La Vecchia C, Negri E, Cecchetti G, Fedele L. Epidemiologic characteristics of women with uterine fibroids: A case-control study. Obstetrics and Gynecology 1988;72(6):853-857. [PubMed: 3186092]

Thoits PA. Stress, coping, and social support processes: Where are we? what next? Journal of Health and Social Behavior 1995:53-79. Spec No. [PubMed: 7560850]

Walker CL, Stewart EA. Uterine fibroids: The elephant in the room. Science (New York, N.Y.) 2005;308(5728):1589-1592.

Wise LA, Palmer JR, Cozier YC, Hunt MO, Stewart EA, Rosenberg L. Perceived racial discrimination and risk of uterine leiomyomata. Epidemiology (Cambridge, Mass.) 2007;18(6):747-757.

Zou G. A modified poisson regression approach to prospective studies with binary data. American Journal of Epidemiology 2004;159(7):702-706. [PubMed: 15033648] 
Table 1

Fibroid Status, Number of and Stress of Major Life Events, and Characteristics of Women by Race, Uterine Fibroids Study, 1996.

\begin{tabular}{|c|c|c|c|c|}
\hline \multirow[t]{2}{*}{ Characteristic } & \multicolumn{2}{|c|}{ Black Women $(\mathrm{n}=556)$} & \multicolumn{2}{|c|}{ White Women $(n=373$} \\
\hline & $\%$ & $\mathbf{n}$ & $\%$ & $\mathbf{n}$ \\
\hline \multicolumn{5}{|l|}{ Uterine Fibroid Status at Interview } \\
\hline Yes & 73.6 & 409 & 49.6 & 185 \\
\hline No & 26.4 & 147 & 50.4 & 188 \\
\hline Number of Life Events ${ }^{1}$, Mean (SD), n & \multicolumn{2}{|c|}{$2.4(2.1), 556$} & \multicolumn{2}{|c|}{$2.1(1.7), 373$} \\
\hline 0 & 19.6 & 109 & 21.5 & 80 \\
\hline 1 & 20.7 & 115 & 20.1 & 75 \\
\hline 2 & 18.5 & 103 & 21.5 & 80 \\
\hline 3 & 13.7 & 76 & 16.9 & 63 \\
\hline 4 or more & 27.5 & 153 & 20.1 & 75 \\
\hline \multicolumn{5}{|l|}{ Stress Intensity } \\
\hline None & 19.2 & 107 & 21.5 & 80 \\
\hline Low & 27.2 & 151 & 23.3 & 87 \\
\hline Medium & 21.2 & 118 & 27.1 & 101 \\
\hline High & 24.1 & 134 & 23.9 & 89 \\
\hline Missing & 8.3 & 46 & 4.3 & 16 \\
\hline Age at Uterine Fibroid Diagnosis (yrs) & \multicolumn{2}{|c|}{$41.4(4.4), 556$} & \multicolumn{2}{|c|}{$42.4(4.4), 373$} \\
\hline $35-39$ & 42.3 & 235 & 35.4 & 132 \\
\hline $40-45$ & 32.9 & 183 & 32.7 & 122 \\
\hline$>45$ & 24.8 & 138 & 31.9 & 119 \\
\hline \multicolumn{5}{|l|}{ Educational Attainment } \\
\hline$\leq$ High school & 20.9 & 116 & 3.2 & 12 \\
\hline Some/junior college & 46.8 & 260 & 7.8 & 29 \\
\hline College degree/plus additional training & 20.9 & 116 & 32.4 & 121 \\
\hline Post graduate degree & 11.5 & 64 & 56.6 & 211 \\
\hline \multicolumn{5}{|l|}{ Adjusted Total Household Income } \\
\hline$<\$ 40,000$ & 52.2 & 290 & 11.3 & 42 \\
\hline$\$ 40,000-\$ 59,999$ & 14.8 & 82 & 11.0 & 41 \\
\hline$\$ 60,000-\$ 99,999$ & 20.0 & 111 & 41.8 & 156 \\
\hline$>\$ 100,000$ & 11.5 & 64 & 35.4 & 132 \\
\hline Missing & 1.6 & 9 & 0.5 & 2 \\
\hline \multicolumn{5}{|l|}{ Occupational Status Longest Held Job } \\
\hline White collar & 35.4 & 197 & 76.4 & 285 \\
\hline Non-white collar & 64.6 & 359 & 23.6 & 88 \\
\hline \multicolumn{5}{|l|}{ No. of Full Term Pregnancies After } \\
\hline \multicolumn{5}{|l|}{ Age 24} \\
\hline 0 & 49.8 & 277 & 62.7 & 234 \\
\hline 1 & 31.3 & 174 & 14.8 & 55 \\
\hline
\end{tabular}




\begin{tabular}{|c|c|c|c|c|}
\hline \multirow[t]{2}{*}{ Characteristic } & \multicolumn{2}{|c|}{ Black Women $(\mathrm{n}=556)$} & \multicolumn{2}{|c|}{ White Women $(n=373$} \\
\hline & $\%$ & $\mathbf{n}$ & $\%$ & $\mathbf{n}$ \\
\hline$\geq 2$ & 18.9 & 105 & 22.5 & 84 \\
\hline \multicolumn{5}{|l|}{ Age at Menarche (yrs) } \\
\hline$<11$ & 12.0 & 64 & 4.0 & 15 \\
\hline 11 & 15.4 & 87 & 15.0 & 56 \\
\hline 12 & 26.8 & 149 & 25.7 & 96 \\
\hline 13 & 23.6 & 132 & 34.9 & 130 \\
\hline 14 & 10.3 & 54 & 11.8 & 44 \\
\hline$>14$ & 12.0 & 67 & 8.3 & 31 \\
\hline Missins & 0.5 & 3 & 0.3 & 1 \\
\hline \multicolumn{5}{|l|}{ Physical Activity } \\
\hline Low $\left(33^{\text {rd }}\right.$ percentile $)$ & 37.2 & 207 & 26.5 & 99 \\
\hline Medium $\left(66^{\text {th }}\right.$ percentile $)$ & 32.6 & 181 & 34.9 & 130 \\
\hline High $\left(83^{\text {rd }}\right.$ percentile) & 15.1 & 84 & 20.4 & 76 \\
\hline Very high $\left(100^{\text {th }}\right.$ percentile $)$ & 14.4 & 80 & 18.0 & 67 \\
\hline Missing & 0.7 & 4 & 1 & 0.3 \\
\hline BMI, mean (SD), n & \multicolumn{2}{|c|}{$30.6(7.9), 556$} & \multicolumn{2}{|c|}{$26.0(6.2), 373$} \\
\hline \multicolumn{5}{|l|}{ Marital Status } \\
\hline Single/never married & 29.1 & 162 & 30.8 & 115 \\
\hline Married/living as married & 42.3 & 235 & 58.2 & 217 \\
\hline Widowed/separated/divorce & 28.4 & 158 & 11.0 & 41 \\
\hline Missing & 0.2 & 1 & 0 & 0 \\
\hline
\end{tabular}

${ }^{1}$ Due to missing values for some women who only answered yes/no to experiencing a life event), the number of women who did not experience any of the 14 life events does not equal the number of women in the 'none' category of stress intensity. 
Table 2

Prevalence Ratio (PR) and 95\% Confidence Interval (CI) for the Association ${ }^{1}$ between the Number of Major Life Events in the Past 12 Months and Fibroid Status by Race, Uterine Fibroid Study, 1996-1999.

\begin{tabular}{|c|c|c|c|c|}
\hline Number of Life Events & Model 1 PR (95\% CI) & Model 2 PR (95\% CI) & Model 3 PR (95\% CI) & Model 4 PR (95\% CI) \\
\hline Black Women & $\mathrm{N}=556$ & $\mathrm{~N}=549$ & $\mathrm{~N}=484$ & $\mathrm{~N}=540$ \\
\hline 1 & $1.1(0.9,1.3)$ & $1.1(0.9,1.3)$ & $1.0(0.9,1.3)$ & $1.1(0.9,1.3)$ \\
\hline 2 & $1.1(0.9,1.2)$ & $1.1(0.9,1.3)$ & $1.1(0.9,1.3)$ & $1.1(0.9,1.3)$ \\
\hline 3 & $1.1(1.0,1.4)$ & $1.1(1.0,1.3)$ & $1.1(0.9,1.3)$ & $1.1(1.0,1.4)$ \\
\hline 4 or more & $1.1(0.9,1.3)$ & $1.1(1.0,1.3)$ & $1.1(0.9,1.2)$ & $1.2(1.0,1.4)$ \\
\hline$P$-value ${ }^{2}$ & 0.62 & 0.64 & 0.94 & 0.48 \\
\hline White Women & $\mathrm{N}=373$ & $\mathrm{~N}=371$ & $\mathrm{~N}=354$ & $\mathrm{~N}=369$ \\
\hline 1 & $1.7(1.2,2.4)$ & $1.7(1.2,2.4)$ & $1.7(1.2,2.5)$ & $1.6(1.1,2.4)$ \\
\hline 2 & $1.6(1.2,2.4)$ & $1.7(1.2,2.4)$ & $1.7(1.2,2.5)$ & $1.6(1.1,2.3)$ \\
\hline 3 & $1.9(1.3,2.7)$ & $1.9(1.3,2.7)$ & $1.8(1.3,2.7)$ & $1.8(1.3,2.6)$ \\
\hline 4 or more & $1.4(1.0,2.1)$ & $1.5(1.0,2.1)$ & $1.5(1.0,2.2)$ & $1.4(0.9,2.1)$ \\
\hline$P$-value ${ }^{2}$ & 0.01 & 0.01 & 0.02 & 0.01 \\
\hline
\end{tabular}

Model 1: Adjusted for age at fibroid diagnosis

Model 2: Model 1 adjusted for age at menarche, BMI, physical activity without chores, No. of Full Term Pregnancies after Age 24

Model 3: Model 2 excluding 17 white women and 65 black women with severe symptoms related to fibroids

Model 4: Model 2 adjusted for education, income, and occupational status

${ }^{1}$ Reference group $=$ no events

2 Bases on Wald Test for type 3 GEE Analysis, two-sided 
Table 3

Prevalence Ratio (PR) and 95\% Confidence Interval (CI) for the Association ${ }^{1}$ between Stress Intensity of Major Life Events (MLE) in the Past 12 Months and Fibroid Status, Uterine Fibroid Study, 1996-1999.

\begin{tabular}{lllll} 
Stress Intensity of MLE & Model $\mathbf{1}$ PR $(\mathbf{9 5} \% \mathbf{C I})$ & Model $\mathbf{2}$ PR $(\mathbf{9 5} \% \mathbf{C I})$ & Model 3 PR $(\mathbf{9 5} \% \mathbf{C I})$ & Model 4 PR $(\mathbf{9 5 \%}$ CI) \\
\hline Black Women & $\mathrm{N}=510$ & $\mathrm{~N}=503$ & $\mathrm{~N}=449$ & $\mathrm{~N}=494$ \\
Low & $1.0(0.9,1.2)$ & $1.1(0.9,1.2)$ & $1.0(0.9,1.2)$ & $1.1(0.9,1.3)$ \\
Medium & $1.1(1.0,1.3)$ & $1.1(1.0,1.3)$ & $1.1(0.9,1.3)$ & $1.1(1.0,1.4)$ \\
High & $1.2(1.0,1.4)$ & $1.2(1.0,1.4)$ & $1.1(1.0,1.4)$ & $1.2(1.0,1.4)$ \\
$P$-value 2 & 0.08 & 0.06 & 0.25 & 0.04 \\
White Women & $\mathrm{N}=357$ & $\mathrm{~N}=355$ & $\mathrm{~N}=339$ & $\mathrm{~N}=353$ \\
Low & $1.8(1.2,2.5)$ & $1.8(1.3,2.5)$ & $1.8(1.3,2.6)$ & $1.7(1.2,2.4)$ \\
Medium & $1.7(1.2,2.4)$ & $1.8(1.2,2.5)$ & $1.8(1.2,2.5)$ & $1.7(1.2,2.4)$ \\
High & $1.5(1.1,2.2)$ & $1.5(1.1,2.2)$ & $1.5(1.0,2.2)$ & $1.5(1.0,2.1)$ \\
$P$-value 2 & 0.01 & 0.01 & 0.01 & 0.01 \\
\hline
\end{tabular}

Model 1: Adjusted for age at fibroid diagnosis

Model 2: Model 1 adjusted for age at menarche, BMI, physical activity without chores, No. of Full Term Pregnancies after Age 24

Model 3: Model 2 excluding 16 white women and 54 black women with severe fibroid related symptoms

Model 4: Model 2 adjusted for education, income, and occupational status

${ }^{1}$ Reference group $=$ No events

2 Based on Wald Test for Type 3 GEE Analysis, two-sided 